\begin{tabular}{|c|c|}
\hline & $\begin{array}{l}\text { International Journal of Trend in Scientific } \\
\text { Research and Development (IJTSRD) }\end{array}$ \\
\hline 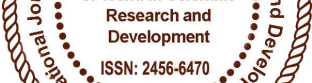 & International Open Access Journal \\
\hline 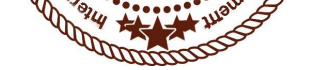 & ISSN No: 2456 - 6470 | www.ijtsrd.com | Volume - 2 | Issue - 2 \\
\hline
\end{tabular}

\title{
Design of Dual Pulsating Latch Flip-Flop (DPLFF) using Novel Pulse Generator
}

\author{
Surbhi Vishwakarma \\ M.Tech Student, (Embedded System \& VLSI Design) \\ Department of Electronics and Communication \\ GGITS, Jabalpur, Madhya Pradesh, India
}

\author{
Dr. Vinod Kapse \\ Principal \& Guide, \\ Department of Electronics and Communication \\ GGITS, Jabalpur, Madhya Pradesh, India
}

\section{ABSTRACT}

In this paper various flip-flop structures have been studied. In all designs to reduce power consumption, the Pulse Generator circuitry should be in build along with the flip-flop itself. If a pulse generator is included along with DPSCRFF structure, power consumption can be reduced. In this work a new design of flip-flop, Double Pulse Latch Flip-flop (DPLFF) is proposed. DPLFF eliminates unnecessary glitches, which consume more power. DPLFF consume less power for same delay as compared with other existing techniques, which is performing one of the fastest known flip-flops. In serial operation as shift register the proposed DPLFF can perform better at the higher frequency. The stacking of transistor in the latch stage cause reduction in subthreshold leakage current $\&$ thus the static power consumption is also less for DPLFF. This is better suited for low power circuits at deep submicron technology where leakages are more dominant.

Keywords: DPLFF, Delay, Power Consumption, Speed, Latch, Flip-flop, Pulse Generator

\section{INTRODUCTION}

The relentless drive toward smaller, faster, and cheaper computing systems has, in large part, been enabled by exponential increases in device density and operating frequency through VLSI technology scaling. This, however, has led to exponential increases in power consumption that has reached the limits of reliability and cost effective cooling. Also, the continuous scaling into the area of nanometer regime introduced issues like signal integrity, design robustness, soft error, \& environmental \& process variability. Furthermore, the issues of power consumption \& robustness only get worse with time. This has created, therefore, a crisis in computer system design that threatens to be a stumbling block to future advancement. Designers of leading-edge computing systems, at any scale, are finding that power consumption $\&$ design robustness are first class constraints, \& must be taken into account at every level of design.

Flip-Flops are critical timing elements in digital circuits \& have a large impact on circuit speed \& power consumption. Consequently, extensive research has been performed to develop fast \& low-power flipflops [1-5] The primary measure of performance of a flip-flop is the minimum Dto- Q delay [1], as it tell about the speed of operations of the flip-flop in each cycle time. Recently, pulsed latch structures have emerged as the fastest known flip-flop structures [1], [2]. By reducing the transparency period of a latch to a narrow window, the latch can operate as a flip-flop with the additional advantage of allowing limited time borrowing across cycle boundaries to reduce sensitivity to clock skew \& jitter. In this process we have designed a new flip-flop called Doubled Pulsed Latch Flip Flop (DPLFF), which is better in terms of 
power \& performance as comparison with Double Pulsed Set Conditional Reset Flip Flop (DPSCRFF).

\section{BACKGROUND - SCALING \& THE CHALLENGES FOR FUTURE COMPUTING}

Integrated circuit technology has advanced tremendously over the past 40 years, as predicted by Moore's Law [5]. Device counts have grown exponentially, from the 2300 transistors of the Intel 4004 processor in 1971 , to the 1700 million transistors of the Intel Dual core Itanium 2 processor in 2006. Simultaneously, clock frequencies have increased exponentially from $0.1 \mathrm{MHz}$ in the Intel 4004 to $3.8 \mathrm{Ghz}$ in Intel Pentium 4's. Historically, \& according to predictions in the International Technology Roadmap for Semiconductors (ITRS) [6], each technology generation, which occur at 2.5-3 year intervals, brings with it a $0.7 \times$ scaling in drawn gate length as well as other layout geometry lengths. The physical gate length follows the same $0.7 \times$ scaling. Assuming a constant die size, this means a $2 \times$ scaling in device count \& a $1.4 \times$ scaling in total transistor width. In addition, the intrinsic switching speed of a transistor increases at roughly $1.5 \times$ per generation. On the other hand, power consumption has been increasing at $20 \%$ per year \& has reached power density limits. At the same time, noise, from many sources, as a fraction of power supply voltage, has increased while noise sensitivity has also increased. These factors, together with increased relative process variation \& environmental variation, have made predictability \& robustness difficult to achieve in new designs. This chapter explains the connection between scaling \& power consumption \& design robustness.

\section{POWER CONSUMPTION}

Power has always been one of the foremost issues in system design. Also, power dissipation, device performance, functionality, battery life, cost, \& size are dependent to each of them. A hand-held device, for example, must be small. There is, therefore, no room for a fan or a large battery. Similarly, a personal computer should be inexpensive; few are willing to pay for exotic cooling technologies. In fact, 15 high performance processors have already reached the power density limit for cost-effective cooling. All these things limit the amount of power a processing chip can burn. The costs of power dissipation extend beyond the power used for computing. Take a data center for example. Firstly, there is, of course, the electricity bill from the computers. Secondly, there is the electricity bill \& maintenance for the air conditioning system, which has to remove the heat due to power dissipation. Finally, thermal concerns dictate a maximum power density of a system; in other words, the more power a system burns, the more space it must occupy. Therefore, we must add in the rent for the space occupied by the system. In all, one account calculates power dissipation at $25 \%$ of the total cost of a data center [7]. Chip power can be divided into two main components: dynamic switching \& static leakage. Dynamic power dissipation, ignoring short-circuit current which is usually a small fraction of total dynamic power, is given by $\mathrm{P}=1 / 2 \mathrm{fCV} 2$, where $\mathrm{C}$ is the average total on-chip capacitance switched per cycle. Ever since the $0.5 \mu \mathrm{m}$ generation, the gate dielectric oxide thickness, supply voltage, \& threshold voltage have scaled with device dimensions by $0.7 \times$ per generation to limit the growth of dynamic power consumption while improving performance. This, however, is only half the power story. The reduction of oxide thickness \& threshold voltage has led to exponential increases in static leakage power. There are six leakage mechanisms in nanometer scale transistors [8], of which the three most significant are subthreshold leakage, gate leakage, \& band-to-band tunneling (BTBT) leakage [9]. These are indicated in Figure 21. Subthreshold leakage is the current flowing from drain to source (or vice versa) when the transistor is nominally off. This current is inversely exponentially proportional to the transistor's threshold voltage \& has therefore grown exponentially. Gate leakage is the current flowing from the gate to the source, drain, or bulk (or vice versa). This is caused by direct tunneling of electrons or holes through the oxide insulator. This current is inversely exponentially proportional to the transistor's oxide thickness, leading to the exponential increase in gate leakage. Band-to-band tunneling is the current flowing through the reverse-biased drain/substrate \& source/substrate junctions. This current is exponentially proportional to the doping concentrations on either side of the junction, which have also increased in scaled devices, leading to the exponential increase in BTBT leakage. Subthreshold leakage was the major component of total leakage at technologies larger than 130nm (drawn gate length).

\section{LITERATURE REVIEW}

[1] Madhav Dasari et-al, "Novel Ultra Low Power Dual Edge Triggered Retention Flip-flop for 
Transiently Powered Systems", IEEE 7th International Advance Computing Conference, 2017.

The paper signifies the need for an improved memory unit that preserves the state of operation for Transiently Powered Systems by offering reduction in the latency for retention and enhance energy efficiency. The authors provided quantitative analysis of different DETFF designs with a retention latch along with SETFF. The designs are evaluated by applying clock and power gating for various levels of voltage operation to verify the suitability for energy harvesting application. A DETFF design based on dynamic design is proposed that excels in all the three levels i.e. super threshold, sub-threshold \& super + sub-threshold when compared with existing DETFF designs.

[2] Andrea Bonetti et-al, "An Overlap-Contention Free True-Single-Phase Clock Dual-Edge-Triggered Flip-Flop", IEEE International Symposium on Circuits and Systems (ISCAS), 2015.

This paper presented a novel dual-edge-triggered flipflop topology to solve the inherent clock-overlap risk in the majority of the previously presented DETFFs. The failure risk due to clock-overlap was demonstrated on a popular DET-TGLM gate, showing an unacceptable error-rate at near-threshold voltages. The proposed fully-static true single-clock-phase DETFF was shown to be fully functional at a similar operating point, under local and global process variations and at a wide range of temperatures. In addition, the proposed cell was found to provide the best performance and energy-efficiency among static DET-FF options.

[3] Nitin Kumar Saini et al, "Low Power Dual Edge Triggered Flip-Flop", IEEE Transactions, Vol.6, Pp978-985, 2014.

In this paper a new technique for pulse generation circuit of Dual Edge Triggered Flip-Flop (DETFF) for low power is presented, which enables the flip-flop to be operated at $1.2 \mathrm{~V}$. By the introduction of a new fast latch \& applying conditional pre-charging, dual-edge triggered flip-flop becomes capable of obtaining low power consumption that has smaller delay. Clockgated sense-amplifier is incorporated to reduce power consumption at low switching activity. Simulation were done in $90 \mathrm{~nm}$ process technology. The advantages of this design are less latency \& low power consumption \& drawbacks is this design is a bulky design.
[4] Kalarikkal Absel et al, "Low-Power Dual Dynamic Node Pulsed Hybrid Flip-Flop Featuring Efficient Embedded Logic", IEEE Transactions On Very Large Scale Integration (VLSI) Systems, Vol. 21, No. 9, September 2013.

In this paper, authors presented two designs of dual edge flip-flops named as, Dual Dynamic Node Hybrid Flip-Flop (DDFF) \& Embedded Logic Module (DDFF-ELM) which is based on DDFF. The proposed designs eliminate the large capacitance present in the precharge node of several state-of-the-art designs by following a split dynamic node structure to separately drive the output pull-up and pull-down transistors. Simulations were done in $90 \mathrm{~nm}$ process technology. It is well suited for modern high performance designs where area, delay-overhead, and power dissipation are of major concern. But this design needed an analysis of the overlap period required to select proper pulse width.

[5] Helga Evangelene et al, "A Novel Low Power Hybrid Flipflop using Sleepy Stack Inverter Pair", IEEE Science and Information Conference-14, London, UK, August, 2014.

This paper presents a low power hybrid flip flop using sleepy stack inverter pair for retaining the logic level till the end of evaluation and pre-charge phase of the flip flop. The sleepy stack inverter pairs are efficient in leakage power reduction and overall power dissipation as the technology scales down to $90 \mathrm{~nm}$ and below. The performance of the proposed flip flop was compared with the conventional Dual Dynamic Node Pulsed Hybrid Flip Flop (DDNPHFF). This design has reduced leakage power with other designs. As the leakage power depends on the input vectors, when the system is idle low leakage input vectors has to be fed as input to the system. The speed of the flip flop is maintained in the proposed flip flop as like in conventional one. The area is the only trade off, i.e. it is a bulky design.

\section{PROPOSED METHODOLOGY}

In design of DPLFF pulse generator is modified, \& the Latch part also modified to eliminates undesired glitch, extra dynamic power, which is caused by preset, \& static power.

\subsection{DPLFF DESIGN}

The two pulses are generated by a local pulse generator to avoid pulse distortions from additional 
pulse buffers \& wiring. The pulse generator can be shared by a few neighboring flip-flops to reduce pulse generator area \& energy overheads. The width of the pulses is controlled by the inverter delay chain. The inverters in the chain can be skewed to control the lengths of $\mathrm{p} 1 \& \mathrm{p} 2$. The width of $\mathrm{p} 2$ determines the transparency window of the latch. To reduce setup \& hold time requirements, $\mathrm{p} 2$ should be made as small as possible. However, if $\mathrm{p} 2$ is too short, the circuit will not function. Detailed simulation at all process corners \& careful control of clock pulse loading will ensure proper functionality.

The conventional way to generate a pair of pulses uses an inverter delay chain as in Fig. 4.10 (a). This design has a large number of intermediate nodes \& thus dissipates a significant amount of energy. Our alternative design reduces the number of intermediate nodes by using an inverter delay chain both to generate $\mathrm{p} 2 \&$ to turn off $\mathrm{p} 1$.

Figure 6.1 shows the design of the DPLFF.-The DPLFF consist a static latch \& pulse generator. Static latch required two pulses p1 \& p2, which are generated from the active clock edge. When data in 0 PM2 is on \& NM1 is off, the pulse p1 will be low for small amount of time with in that time output node will be charged. When the input data is ' 1 ' PM2 is off \& NM1 is on pulse $\mathrm{p} 2$ will be high for small time \& within that time output node will be discharged. In the Figure 6.2 operation of DPLFF is shown, whenever the output node is to be in low sate it remains low unlike DPSCRFF because of PM2 is off.

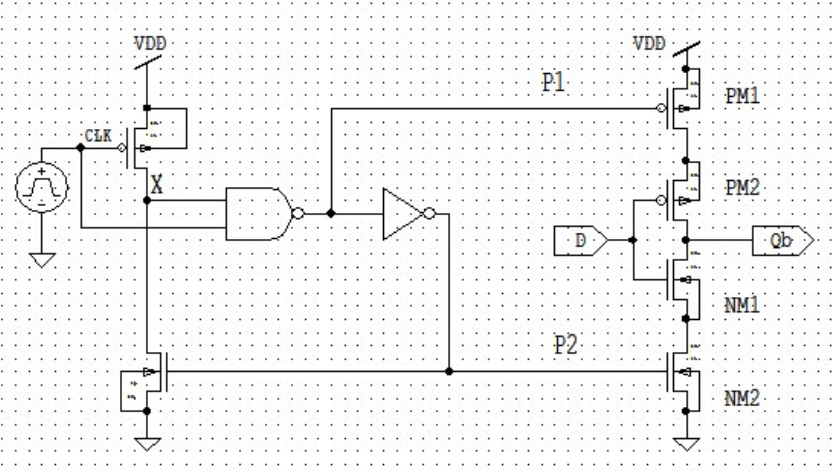

Figure 1: DPLFF using Novel Pulse Generation

The DPLFF has symmetric properties pulse p1 low \& p2 high almost at same time the input should be should not change during this time. But the width of the pulse $\mathrm{p} 1 \& \mathrm{p} 2$ is less than that of the DPSCRFFs pulse $\mathrm{p} 1 \& \mathrm{p} 2$. In DPLFF NMOS in the clock generator is going to switch on after one inverter delay where as in DPSCRFF after 3 inverter delay. This makes the DPLFFs pulse ( 1 \& $\&$ p) width less than that of DPSCRFFs pulse ( 1 \& $\mathrm{p} 2)$. The propagation delay of required in the combinational circuit is also less than DPSCRFF. The maximum clock frequency also increases in shift register when DPLFF is used.

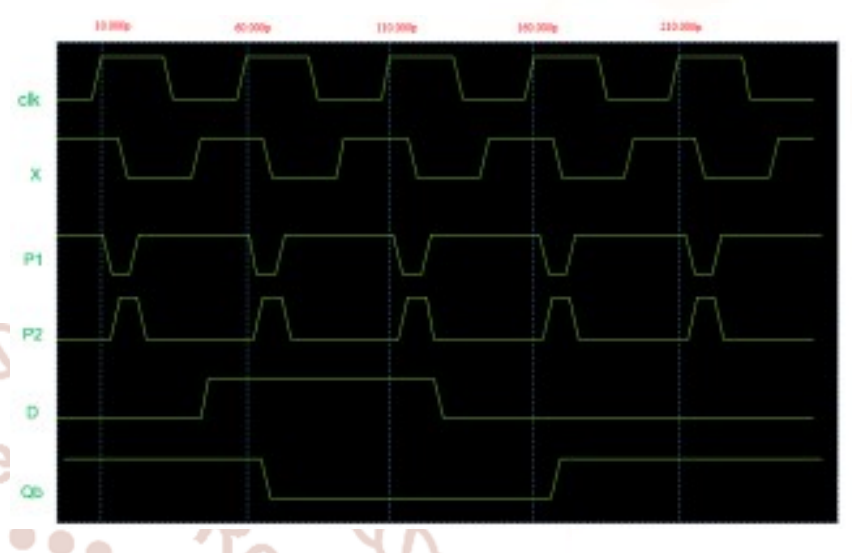

Figure 2: DPLFF Operation Waveforms

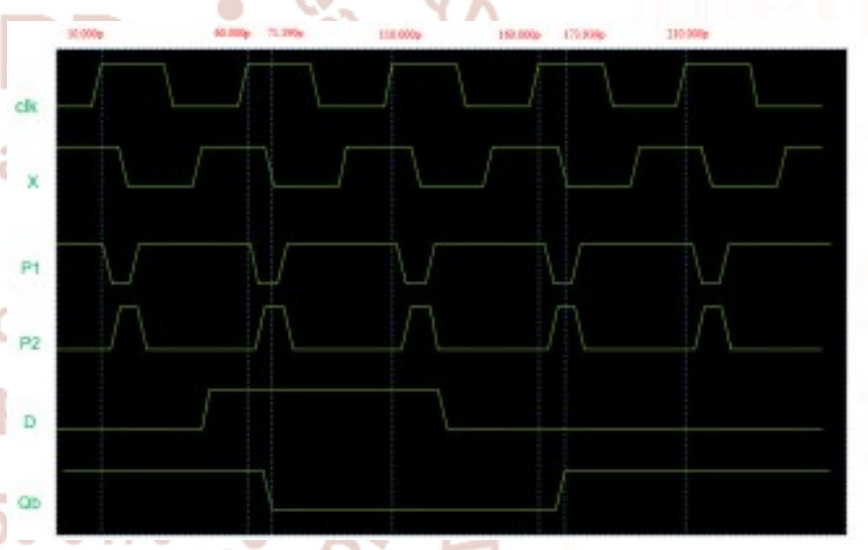

Figure 3: DPLFF Delay Analysis

V. SIMULATION RESULTS

The simulation of the proposed design is done using transient analysis using PTM $65 \mathrm{~nm}$ in HSPICE at $1 \mathrm{GHZ}$ clock frequency the results are tabulated in Table $1 \&$ Table 2 . DPLFF is designed \& simulated by using multi threshold, in the latch part we used low $\mathrm{V}_{\text {th }}$ \& high $\mathrm{V}_{\text {th }}$ everywhere else. The simulation result shows that DPLFF performance is better than other existing techniques in terms of power dissipation in all situations. The static power reduction is obvious when clock \& data are set to zero. The static power saving is considerable \& considering the fact that many flip-flops are used in circuit over all power saving will be large for nanometer designs. The power reduction is achieved by removing two inverters, where static power also reduced in latch by stacking two PMOS (PM1, PM2). Overall power dissipation is 
reduced as compared with DPSCRFF. From the timing analysis shows that DPLFF requires less time when output is changed from ' 1 ' to ' 0 '. However, DPSCRFF delay is zero to change the output from ' 0 ' to ' 1 ' because preset of output node before arrival of input. However, the clock frequencies required for flip-flop will be decided by logic level changes from either side. Thus the operational frequencies for DPLFF will be higher compared to DPSCRFF [18].

\begin{tabular}{|l|c|}
\hline \multicolumn{1}{|c|}{ Condition } & Proposed DPLFF \\
\hline 1. Output is altering ' $\mathbf{1}$ ' to ' 0 ' & $2.788 \mathrm{u}$ watt \\
\hline 2. Output is altering ' 0 ' to ' $\mathbf{~ '}$ & $2.832 \mathrm{u}$ watt \\
\hline 3. Output is constant ' $\mathbf{0}$ ' & $2.163 \mathrm{u}$ watt \\
\hline 4. Output is constant ' $\mathbf{1}$ ' & $1.920 \mathrm{u}$ watt \\
\hline 5. Clk ='0' $\mathbf{D}=$ '0' & $0.1676 \mathrm{u}$ watt \\
\hline
\end{tabular}

Table 1: Power consumption of Proposed DPLFF

\begin{tabular}{|c|c|c|}
\hline Condition & Delay (p-sec) & $\begin{array}{c}\text { Average Delay } \\
\text { CLK-Qb } \\
\text { (p-sec) }\end{array}$ \\
\cline { 1 - 2 } Output changes ' 1 ' to ' 0 ' $\boldsymbol{T}_{p L-H}$ & 11.19 & \multirow{2}{*}{12.56} \\
\cline { 1 - 2 } Output changes ' 0 ' to ' 1 ' $\boldsymbol{T}_{p H-L}$ & 13.94 & \\
\hline
\end{tabular}

Table 2: Delay Performance of Proposed DPLFF

\begin{tabular}{|l|c|c|}
\hline \multicolumn{1}{|c|}{ Flip-flop } & Number of Transistors & Data driving Power $(\boldsymbol{u W})$ \\
\hline SDFF & 23 & 4.56 \\
\hline HLFF & 20 & 5.21 \\
\hline PowerPC 603 & 22 & 16.13 \\
\hline CDMFF & 22 & 13.30 \\
\hline DDFF-ELM [4] & 22 & 3.18 \\
\hline Proposed DPLFF & 16 & 2.788 \\
\hline
\end{tabular}

Table 3 Comparison of Power Consumption

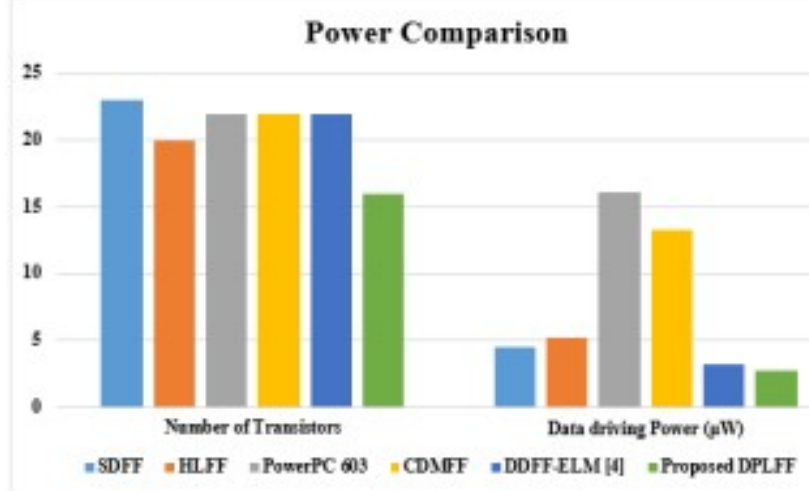

Figure 4 Graph of Power Performance \& No. of transistors

From the table 3 it can be clearly seen that this proposed design has less number of gates used, due to which the average power consumption is also reduced, as compared to other designs. This design has improved power consumption performance with [4], in which 22 transistors were used, but if compared with the ratio of number of transistors \& power consumption, the proposed design is consuming more power.

\begin{tabular}{|c|c|c|c|}
\hline \multirow[t]{2}{*}{ Name of the Circuit } & \multicolumn{2}{|c|}{ Delay (p-sec) } & \multirow{2}{*}{$\begin{array}{c}\text { Average Delay CLK- } \\
Q_{b}(\mathrm{p}-\mathrm{sec})\end{array}$} \\
\hline & $T_{n L-n}$ & $T_{p w-6}$ & \\
\hline SETFF [1] & 75.64 & 92.18 & 83.91 \\
\hline DEIFF 1 & 171.2 & 167.5 & 169.4 \\
\hline DEIFF 2 & 58.27 & 56.28 & 57.28 \\
\hline PDETFF & 71.52 & 92.29 & 81.90 \\
\hline Propesed DPL.FF & 11.19 & 13.94 & 12.56 \\
\hline
\end{tabular}

Table 6.4 Comparison of Delay Performance

The proposed design has better delay performance with respect to other existing designs. It takes only $11.19 \mathrm{p}$-sec when Output changes ' 1 ' to ' 0 ' i.e. $T_{p L-H}$ \& $13.94 \mathrm{p}$-sec when Output changes ' 0 ' to ' 1 ' $T_{p H-L}$. The average delay is $12.56 \mathrm{p}$-sec for the proposed design.

\section{DELAY COMPARISON}

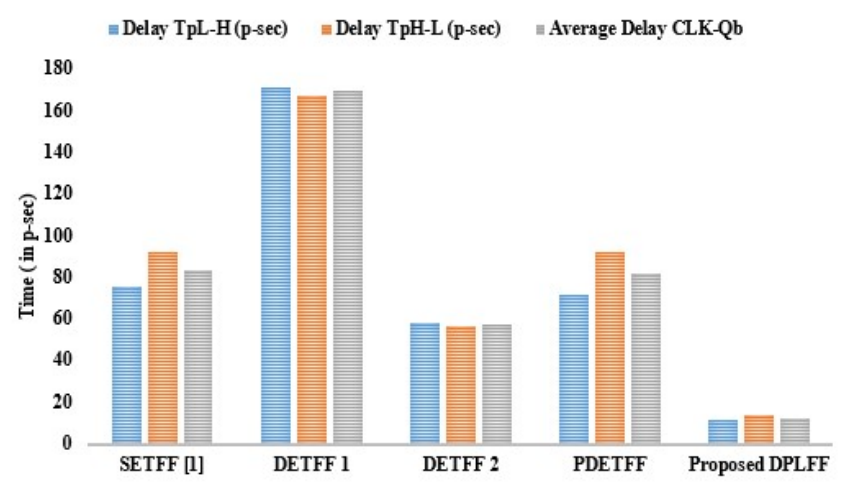

Figure 5: Graph of Delay Performance

\section{CONCLUSION}

In this paper various flip-flop structures has been studied. In all designs to reduce power consumption, the Pulse Generator circuitry should be in build along with the flip-flop itself. If a pulse generator is included along with DPSCRFF structure, power consumption can be reduced In this work a new design of flip-flop, Double Pulse Latch Flip-flop (DPLFF) is proposed. DPLFF eliminates unnecessary glitches, which consume more power. DPLFF consume less power for same delay as compared with other existing techniques, which is performing one of the fastest known flip-flops. In serial operation as 
shift register the proposed DPLFF can perform better at the higher frequency. The stacking of transistor in the latch stage cause reduction in subthreshold leakage current $\&$ thus the static power consumption is also less for DPLFF. This is better suited for low power circuits at deep submicron technology where leakages are more dominant

\section{REFERENCES}

1) Madhav Dasari et-al, "Novel Ultra Low Power Dual Edge Triggered Retention Flip-flop for Transiently Powered Systems", IEEE 7th International Advance Computing Conference, 2017.

2) Andrea Bonetti et-al, "An Overlap-Contention Free True-Single-Phase Clock Dual-EdgeTriggered Flip-Flop", IEEE International Symposium on Circuits and Systems (ISCAS), 2015.

3) Nitin Kumar Saini et al, "Low Power Dual Edge Triggered Flip-Flop", IEEE Transactions, Vol.6, Pp. 978-985, 2014.

4) Kalarikkal Absel et al, "Low-Power Dual Dynamic Node Pulsed Hybrid Flip-Flop Featuring Efficient Embedded Logic", IEEE Transactions On Very Large Scale Integration (VLSI) Systems, Vol. 21, No. 9, September 2013.

5) Helga Evangelene et al, "A Novel Low Power Hybrid Flip-Flop using Sleepy Stack Inverter Pair", IEEE Science and Information Conference2014, London, UK, August, 2014.

6) C. S, Kim et al, "Presetting pulse based flipflop," IEEE International Symposium Circuits Systems (ISCAS 2008),, Pp. 588-591, May 2008.

7) C.C. Yu et al, "Design Of Low-Power Double Edge-Triggered Flip-Flop Circuit," 2nd IEEE Conference on Industrial Electronics Applications (ICIEA 2007), Pp. 2054-2057, May 2007.

8) P. Zhao et al, "Low-power clock branch sharing double edge-triggered flip-flop," IEEE Transactions Very Large Scale Integrated (VLSI) Systems, Vol-15, Issue-3, Pp. 338-345, March 2007.

9) Saibal Mukhopadhyay et al, "Accurate estimation of total leakage in nanometer-scale bulk CMOS circuits based on device geometry and doping profile. IEEE Transactions of Computer-Aided Design of Integrated Circuits and Systems, Vol24, Issue 3, Pp. 363-381, March 2005.

10) A. Agarwal et al, "Leakage power analysis and reduction: models, estimation and tools. IEEE
Proceeding of Computer Digital Technology, Vol152, Issue 3, Pp. 353-368, May 2005.

11) M. W. Phyu et al, "A low-power static dual edge triggered flip-flop using an output controlled discharge configuration," in Proc. IEEE International Symposium Circuits Systems (ISCAS 2005), Vol-3, Pp. 2429-2432, May 2005.

12) G. Aliakbar et al "Dual-Edge Triggered Static Pulsed Flip-Flops," in Proc. 18th International Conference of VLSI Design 2005, Pp. 846-849, Jan. 2005.

13) Samuel K. H. Fung et al, " $65 \mathrm{~nm}$ CMOS high speed, general purpose and low power transistor technology for high volume foundry application" In Symposium of VLSI Tech., Pp. 92-93, 2004.

14) Rajeev R. Rao et al, "Parametric yield estimation considering leakage variability", In DAC, Pp. 442-447, June 2004.

15) P. Zhao et al, "High Performance And LowPower Conditional Discharge Flip-Flop," IEEE Transactions Very Large Scale Integrated (VLSI) Systems,Vol-12, Issue-5, Pp. 477-484, May 2004.

16) Kaushik Roy et al, "Leakage current mechanisms and leakage reduction techniques in deep submicrometer CMOS circuits", Proceeding IEEE, Vol-91, Issue-2, Pp.305-327, February 2003.

17) Saibal Mukhopadhyay et al, "Modeling and estimation of total leakage current in nano-scaled CMOS devices considering the effect of parameter variation", In ISLPED, Pp. 172-175, 2003.

18) Albert Ma et-al, "A Double-Pulsed SetConditional-Reset Flip-Flop”, MIT Laboratory for Computer Science, 2003.

19) B. S. Kong et al "Conditional capture flip-flop for statistical power reduction," IEEE Journal of Solid-State Circuits, Vol-36, Issue-8, Pp. 1263- 1271, August 2001. 